Helsinki University of Technology Institute of Mathematics Research Reports

Teknillisen korkeakoulun matematiikan laitoksen tutkimusraporttisarja

Espoo 2003

A460

\title{
NUMERICAL TAYLOR EXPANSIONS FOR INVARIANT MANIFOLDS
}

Timo Eirola Jan von Pfaler 

Helsinki University of Technology Institute of Mathematics Research Reports

Teknillisen korkeakoulun matematiikan laitoksen tutkimusraporttisarja

Espoo 2003

A460

\title{
NUMERICAL TAYLOR EXPANSIONS FOR INVARIANT MANIFOLDS
}

\author{
Timo Eirola Jan von Pfaler
}

Dedicated to Gerhard Wanner on the occasion of his $60^{\prime}$ th birthday. 
Timo Eirola and Jan von Pfaler: Nmerical Taylor expansions for invariant manifolds; Helsinki University of Technology Institute of Mathematics Research Reports A460 (2003).

\begin{abstract}
We consider numerical computation of Taylor expansions of invariant manifolds around equilibria of maps and flows. These are obtained by writing the corresponding functional equation in a number of points, setting up a nonlinear system of equations and solving that using a simplified Newton's method. This approach will avoid symbolic or explicit numerical differentiation. The linear algebra issues of solving the resulting Sylvester equations are studied in detail.
\end{abstract}

AMS subject classifications: 65Q05 65P, 37M, (secondary) 65P30, 65F20, 15A69

Keywords: numerical approximation of invariant manifolds, multivariate polynomial, bifurcation, Taylor expansion

ISBN 951-22-6504-4

ISSN 0784-3143

HUT Mathematics, 2003

Helsinki University of Technology

Department of Engineering Physics and Mathematics

Institute of Mathematics

P.O. Box 1100, 02015 HUT, Finland

email:math@hut.fi http://www.math.hut.fi/ 


\section{Introduction}

The stable, center, and unstable manifolds of equilibria of dynamical systems are important objects in many applications. This is mainly because the corresponding lower dimensional systems can be studied by obtaining the reduced dynamic on these manifolds.

In this paper we will study Taylor expansions for these smooth manifolds. Such expansions have been studied e.g. in works [Sim89], [Kuz95], and [BK98]. Here, by setting up a system of nonlinear equations we will obtain numerical approximations for the Taylor coefficients, but avoid (symbolic or direct numerical) differentiation.

First, in Section 2 we look at the equations that the functions describing the manifolds of interest need to satisfy. Section 3 sets up the necessary theoretical tools and equations for the coefficients of the Taylor series in the traditional way. The present approach simplifies previous presentations via use of symmetries. In Section 4 we give a new approach by setting up a nonlinear system of equations for all the coefficients of interest at once, and this way avoid differentiation of the system equations. Finally, in Section 5 we test our approach in four examples: a simple map, a system of coupled Tshebyshev maps, the Lorenz system, and a PDE system, called the brusselator. In the latter example we also demonstrate the use of these expansions in bifurcation analysis.

\section{Equations for local invariant manifolds}

Consider a discrete dynamical system $x^{k+1}=\phi\left(x^{k}\right)$, where $\phi: \mathbb{R}^{n} \rightarrow \mathbb{R}^{n}$ is a diffeomorphism of class $C^{r}$ and let $p$ be its fixed point. Split the spectrum $\sigma(D \phi(p))=\Lambda \cup \widetilde{\Lambda}, \Lambda \cap \widetilde{\Lambda}=\emptyset$, such that $\bar{\Lambda}=\Lambda$ and let $E \oplus \widetilde{E}=\mathbb{R}^{n}$ be the corresponding splitting to invariant subspaces. Then under certain gap condition on $\Lambda, \widetilde{\Lambda}$ there exists a local smooth $\phi$-invariant manifold $W$ such that $T_{p} W=E$ (see e.g. [Shu87]).

Important special cases of such manifolds are the following

- for $\Lambda=\{\lambda \in \sigma(D \phi(p))|| \lambda \mid<1\} W=W_{p}^{s}$ is the local stable manifold of $p$,

- for $\Lambda=\{\lambda \in \sigma(D \phi(p))|| \lambda \mid=1\} W=W_{p}^{c}$ is the center manifold of $p$,

- for $\Lambda=\{\lambda \in \sigma(D \phi(p))|| \lambda \mid>1\} W=W_{p}^{u}$ is the local unstable manifold of $p$.

Let the columns of matrices $U \in \mathbb{R}^{n \times d}$ and $\widetilde{U} \in \mathbb{R}^{n \times(n-d)}$ span $E$ and $\widetilde{E}$, respectively, and put $\left[\begin{array}{ll}V & \widetilde{V}\end{array}\right]^{T}=\left[\begin{array}{ll}U & \widetilde{U}\end{array}\right]^{-1}$. We set local coordinates via $x=$

\footnotetext{
${ }^{0}$ Version: October 27, 2003
} 
$p+U s+\widetilde{U} \widetilde{s}$. Then, in these coordinates, we write $\phi$ as

$$
\left[\begin{array}{l}
\eta(s, \widetilde{s}) \\
\widetilde{\eta}(s, \widetilde{s})
\end{array}\right]=\left[\begin{array}{l}
V^{T} \\
\widetilde{V}^{T}
\end{array}\right][\phi(p+U s+\widetilde{U} \widetilde{s})-p] .
$$

Since $W$ is tangent to $E$ at $p$, it can locally be written (in $(s, \widetilde{s})$-coordinates) as a graph of a function $g: \mathbb{R}^{d} \rightarrow \mathbb{R}^{n-d}$ by $W=\{(s, g(s))\}$ with $s$ varying in a neighbourhood of the origin. By invariance, $g$ has to satisfy the following functional equation:

$$
\widetilde{\eta}(s, g(s))=g(\eta(s, g(s))),
$$

with $g(0)=0, D g(0)=0$. Then, restricted to $W$, the dynamical system is

$$
s^{k+1}=\eta\left(s^{k}, g\left(s^{k}\right)\right) \text {. }
$$

Similarly, consider an equilibrium $f(p)=0$ of an autonomous differential equation $\dot{x}=f(x)$, where $f$ is a smooth vector field. If we split the spectrum of $D f(p)$, then under suitable gap condition (see: [Har64], [Shu87]) we obtain a smooth invariant manifold corresponding to this splitting. Again, we take local coordinates as above, write

$$
\left[\begin{array}{l}
\eta(s, \widetilde{s}) \\
\widetilde{\eta}(s, \widetilde{s})
\end{array}\right]=\left[\begin{array}{c}
V^{T} \\
\widetilde{V}^{T}
\end{array}\right] f(p+U s+\widetilde{U} \widetilde{s}),
$$

and the manifold as a graph of a function: $W=\{(s, g(s))\}$. Now, by invariance, $g$ satisfies the following first order quasilinear partial differential equation

$$
\widetilde{\eta}(s, g(s))=D g(s) \eta(s, g(s))
$$

with $g(0)=0, D g(0)=0$. The system, restricted to $W$, is now

$$
\dot{s}=\eta(s, g(s)) \text {. }
$$

The usual cases of stable, center, and unstable manifold correspond now to the splittings of the real parts of the eigenvalues according to $<0,=0$, and $>0$.

\section{Taylor expansions}

Since $W$ is the graph of a smooth function $g$, it is natural to try to approximate $g$ by the Taylor expansion. In this section we describe - both for the discrete and the differential system - the equations that the coefficients of the Taylor expansion need to satisfy, and show that they are uniquely solvable if certain nonresonance conditions are satisfied. These conditions are weaker than the gap condition for the existence of the smooth manifold. This part contains known results that are here written using some new tools and notation, which will be 
useful later. For references, see e.g. [Sim89], [Kuz95], and [BK98]. For example, by using symmetries from the very beginning, our approach becomes simpler and the multilinear Sylvester equations of [BK98] are reduced to usual Sylvester equations. We also describe, for later use, two ideas of paper [BK98] for solving the Sylvester equations.

\section{Discrete system}

Consider the functional equation (2.1):

$$
\widetilde{\eta}(s, g(s))=g(\eta(s, g(s)))
$$

and write $g$ as formal power series

$$
g(s)=\sum_{k \geq 2} \gamma_{k}[s]^{k} .
$$

Here each coefficient $\gamma_{k}$ is a symmetric ${ }^{1} k$-linear map $\mathbb{R}^{d \times k} \mapsto \mathbb{R}^{n-d}$ and $[s]^{k}=(s, \ldots, s) \in \mathbb{R}^{d \times k}$. Equivalently, see $[\operatorname{Lan} 71], p_{k}(s)=\gamma_{k}[s]^{k}$ is a degree $k$ homogeneous polynomial of $d$ variables with values in $\mathbb{R}^{n-d}$. Similarly, write

$$
\begin{aligned}
& \eta(s, \widetilde{s})=\sum_{i+j \geq 1} \mu_{i, j}[s]^{i}[\widetilde{s}]^{j} \\
& \widetilde{\eta}(s, \widetilde{s})=\sum_{i+j \geq 1} \widetilde{\mu}_{i, j}[s]^{i}[\widetilde{s}]^{j}
\end{aligned}
$$

where $\mu_{i, j}: \mathbb{R}^{d \times i} \times \mathbb{R}^{(n-d) \times j} \mapsto \mathbb{R}^{d}$ and $\widetilde{\mu}_{i, j}: \mathbb{R}^{d \times i} \times \mathbb{R}^{(n-d) \times j} \mapsto \mathbb{R}^{n-d}$ are $i+j$-linear and symmetric with respect to $s$ and $\widetilde{s}$-parts separately. Then (3.1) reads

$$
\begin{aligned}
& \sum_{i+j \geq 1} \tilde{\mu}_{i, j}[s]^{i}\left[\sum_{k \geq 2} \gamma_{k}[s]^{k}\right]^{j}= \\
& =\sum_{k \geq 2} \gamma_{k}\left[\sum_{i+j \geq 1} \mu_{i, j}[s]^{i}\left[\sum_{l \geq 2} \gamma_{l}[s]^{l}\right]^{j}\right]^{k} .
\end{aligned}
$$

Since $E, \widetilde{E}$ are the $D \phi(p)$-invariant subspaces, we have $\mu_{0,1}=0$ and $\widetilde{\mu}_{1,0}=0$. Denote $A=\mu_{1,0}$ and $\widetilde{A}=\widetilde{\mu}_{0,1}$. These have spectra $\Lambda$ and $\widetilde{\Lambda}$, respectively. Write (3.4) as

$$
\begin{aligned}
& \widetilde{A}\left(\gamma_{2}[s]^{2}+\gamma_{3}[s]^{3}+\ldots\right)+\widetilde{\mu}_{2,0}[s]^{2}+\widetilde{\mu}_{1,1}\left(s, \gamma_{2}[s]^{2}+\ldots\right)+\ldots \\
& =\gamma_{2}\left[A s+\mu_{2,0}[s]^{2}+\mu_{1,1}\left(s, \gamma_{2}[s]^{2}+\ldots\right)+\ldots\right]^{2}+ \\
& \quad+\gamma_{3}\left[A s+\mu_{2,0}[s]^{2}+\ldots\right]^{3}+\ldots
\end{aligned}
$$

Comparing the powers of $s$ gives

$$
\begin{aligned}
& \widetilde{A} \gamma_{2}[s]^{2}+\widetilde{\mu}_{2,0}[s]^{2}=\gamma_{2}[A s]^{2}, \\
& \widetilde{A} \gamma_{3}[s]^{3}+\widetilde{\mu}_{1,1}\left(s, \gamma_{2}[s]^{2}\right)+\widetilde{\mu}_{3,0}[s]^{3}=2 \gamma_{2}\left(A s, \mu_{2,0}[s]^{2}\right)+\gamma_{3}[A s]^{3},
\end{aligned}
$$

\footnotetext{
${ }^{1}$ Symmetric: $\gamma_{k}\left(s^{\pi(1)}, \ldots, s^{\pi(k)}\right)=\gamma_{k}\left(s^{1}, \ldots, s^{k}\right)$ for every permutation $\pi:\{1, \ldots, k\} \mapsto$ $\{1, \ldots, k\}$
} 
These equations can be solved recursively: the equation for $\gamma_{k}$ is of the form

$$
\widetilde{A} \gamma_{k}[s]^{k}-\gamma_{k}[A s]^{k}=\text { given , }
$$

where the right hand side depends only on $\gamma_{2}, \ldots, \gamma_{k-1}$.

For fixing a symmetric $k$-linear map uniquely, it is sufficient to give its polar form, i.e. the values of the map on the diagonal $[s]^{k}=(s, \ldots, s) \in \mathbb{R}^{d \times k}$, see [CH82, Chapter 2.1].

Now, for a multi-index $u \in \mathbb{N}_{0}^{d}$ define $s^{u}=\prod_{i=1}^{d} s_{i}^{u_{i}},|u|=\sum_{i} u_{i}=\operatorname{deg} s^{u}$, and $u !=\prod_{i} u_{i}$ !, Let ${ }^{2} \quad c_{u}=\sqrt{|u| ! / u !}$. Denote by $\mathbb{P}_{k}$ the space of homogeneous polynomials of $d$ variables and degree $k$. It has dimension $\left(\begin{array}{c}k+d-1 \\ d-1\end{array}\right)$. Let

$$
\left\{c_{u} s^{u}|| u \mid=k\right\}=\left\{s_{1}^{k}, \sqrt{k} s_{1}^{k-1} s_{2}, \ldots, s_{d}^{k}\right\}
$$

be the lexicographically ordered monomial basis of $\mathbb{P}_{k}$. The basis is scaled for convenience. Collecting the elements of the set (3.6) into a vector we can write

$$
\gamma_{k}[s]^{k}=\Gamma_{k} \widehat{s}^{k}
$$

where

$$
\Gamma_{k} \in \mathbb{R}^{(n-d) \times \operatorname{dim} \mathbb{P}_{k}} \quad \text { and } \quad \widehat{s}^{k}=\left[\begin{array}{c}
s_{1}^{k} \\
\sqrt{k} s_{1}^{k-1} s_{2} \\
\vdots \\
s_{d}^{k}
\end{array}\right] .
$$

Then (3.5) becomes

$$
\widetilde{A} \Gamma_{k}-\Gamma_{k} B_{A, k}=\text { given , }
$$

where the transpose of $B_{A, k}$ is the matrix of the linear map $L_{A}(p)(s)=p(A s)$ with respect to the monomial basis. Sylvester equation (3.8) is uniquely solvable if $\sigma(\widetilde{A}) \cap \sigma\left(B_{A, k}\right)=\emptyset$. The following lemma helps us to infer this. For a more general result, see Thm. 3.2 of [BK98].

Lemma 3.1. Let $\underline{\lambda}=\left[\begin{array}{llll}\lambda_{1} & \lambda_{2} & \ldots & \lambda_{d}\end{array}\right]$ be a vector consisting of the eigenvalues of $A$. Then

$$
\sigma\left(B_{A, k}\right)=\sigma(A)^{\cdot k}:=\left\{\underline{\lambda}^{j}\left|j \in \mathbb{N}_{0}^{d},\right| j \mid=k\right\},
$$

i.e., the set of all $k$-fold products of elements of $\sigma(A)$.

Proof. Assume first that $A$ is diagonalizable: $A=X \Lambda X^{-1}, \Lambda=\operatorname{diag}(\underline{\lambda})$. Then

$$
\begin{aligned}
L_{A}(p)(s) & =p\left(X \Lambda X^{-1} s\right)=L_{X}(p)\left(\Lambda X^{-1} s\right) \\
& =L_{\Lambda}\left(L_{X}(p)\right)\left(X^{-1} s\right)=\left(L_{X}^{-1} \circ L_{\Lambda} \circ L_{X}\right)(p)(s) .
\end{aligned}
$$

Hence $L_{A}$ is similar to $L_{\Lambda}$. Clearly the monomials are eigenfunctions of $L_{\Lambda}$ and

$$
\sigma\left(L_{\Lambda}\right)=\left\{\underline{\lambda}^{j}|| j \mid=k\right\} .
$$

Hence (3.9) holds, if $A$ is diagonalizable. Then, since diagonalizable matrices are dense and both sides of (3.9) depend continuously on $A$, they will be equal for all $A$.

\footnotetext{
${ }^{2}|u| ! / u$ ! is often called the multinomial coefficient.
} 
By Lemma 3.1 the Taylor expansion of $g$ exists (formally) up to terms of order $k$ if the nonresonance condition

$$
\sigma(\widetilde{A}) \cap \sigma(A)^{\cdot j}=\emptyset, j=1, \ldots, k
$$

is satisfied. For example, in the case of (strong) stable manifold, $\sigma(A)$ contains all eigenvalues with absolute value less than one. Then (3.10) is satisfied for all $k$.

In the following proposition we have collected some properties of the matrix $B_{A, k}$ that will become useful in the end of the section. For the proposition, consider $\mathbb{P}_{k}^{d}$, i.e. the set of the $\mathbb{R}^{d}$ valued homogenous polynomials of degree $k$, and let its basis be

$$
\left\{c_{u} s^{u} e_{j}|| u \mid=k, j=1, \ldots, d\right\} .
$$

Define a linear map $\Psi: \mathbb{P}_{k}^{d} \mapsto \mathbb{P}_{k+1}$, via the basis as $\left(s^{u} e_{j}\right) \mapsto s^{u} s_{j}$, and let $P_{k}$ be the matrix of this mapping. Note that $P_{k}$ is rather simple, every column of $P_{k}$ only has one element, and it is nonzero and positive.

Proposition 3.2. The matrices $B_{A, k}$ satisfy the recursion

$$
B_{A, k+1}=P_{k}\left(B_{A, k} \otimes A\right) P_{k}^{T} \text { with } B_{A, 0}=1,
$$

for any $k \in \mathbb{N}_{0}$. Further, $B_{A^{*}, k}=\left(B_{A, k}\right)^{*}$, and $B_{A, k}$ is upper (respectively lower) triangular if $A$ is.

It follows from the Proposition and the fact $B_{A, k}^{-1}=B_{A^{-1}, k}$, that $B_{A, k}$ is unitary if $A$ is.

Proof. Fix $k \in \mathbb{N}_{0}$. Denote by $\left\{e_{u}|| u \mid=k\right\}$ the standard basis of $\mathbb{R}^{\operatorname{dim} \mathbb{P}_{k}}$ with the lexicographic ordering. Then

$$
\left\{e_{u} \otimes e_{j}|| u \mid=k, j=1, \ldots, d\right\}
$$

is the basis for the coordinates of the elements of $\mathbb{P}_{k}{ }^{d}$.

We first show that the matrices $B_{A, k}$ can be formed recursively. Clearly $B_{A, 0}=1$. Denote by $\widetilde{L}_{A}$ the componentwise evaluation ${ }^{3}$ of $L_{A}$. Then for any $u,|u|=k$ and $j \in\{1,2, \ldots, d\}$,

$$
\left(L_{A} \circ \Psi\right)\left(s^{u} e_{j}\right)=L_{A}\left(s^{u+e_{j}}\right)=(A s)^{u+e_{j}}
$$

and on the other hand,

$$
\begin{aligned}
&\left(\Psi \circ A^{T} \circ \widetilde{L}_{A}\right)\left(s^{u} e_{j}\right)=\left(\Psi \circ A^{T}\right)\left((A s)^{u} e_{j}\right)=\Psi\left((A s)^{u}\left(A^{T} e_{j}\right)\right) \\
&=(A s)^{u} \sum_{i} A_{j i} s_{i}=(A s)^{u}(A s)^{e_{j}}=(A s)^{u+e_{j}} . \\
&{ }^{3} \widetilde{L}_{A}\left(p_{1}, p_{2}, \ldots, p_{d}\right)=\left(L_{A}\left(p_{1}\right), \ldots, L_{A}\left(p_{d}\right)\right) .
\end{aligned}
$$


Thus $L_{A} \circ \Psi=\Psi \circ A^{T} \circ \widetilde{L}_{A}$ and by the association of $c_{u} s^{u} e_{j}$ with $e_{u} \otimes e_{j}$, we get

$$
B_{A, k+1}^{T} P_{k}=P_{k}\left(I \otimes A^{T}\right)\left(B_{A, k}^{T} \otimes I\right)=P_{k}\left(B_{A, k}^{T} \otimes A^{T}\right) .
$$

Clearly $\Psi$ is onto, so that $P_{k} P_{k}^{T}$ is invertible. Multiplying $B_{A, k+1}^{T} P_{k}$ with $P_{k}^{T}\left(P_{k} P_{k}^{T}\right)^{-1}$ gives

$$
B_{A, k+1}^{T}=P_{k}\left(B_{A, k}^{T} \otimes A^{T}\right) P_{k}^{T}\left(P_{k} P_{k}^{T}\right)^{-1} .
$$

Let $w$ and $w^{\prime}$ be such that $\left|w^{\prime}\right|=|w|=k+1$. By definition of $\Psi$, if $e_{j}+v=w$ then $e_{w}^{T} P_{k}\left(e_{v} \otimes e_{j}\right)=c_{v} / c_{w}=\sqrt{w_{j} /(k+1)}$, and otherwise zero. Then

$$
P_{k}^{T} e_{w}=\sum_{(v, j) \in \mathcal{I}_{w}} \sqrt{\frac{w_{j}}{k+1}} e_{v} \otimes e_{j}
$$

where $\mathcal{I}_{w}=\left\{(v, j)\left|w=v+e_{j},\right| v \mid=k, j \in 1, \ldots, d\right\}$. If $w \neq w^{\prime}$, then $e_{w}^{T} P_{k} P_{k}^{T} e_{w^{\prime}}=\left(P_{k}^{T} e_{w}\right)^{T}\left(P_{k}^{T} e_{w^{\prime}}\right)=0$. And

$$
e_{w}^{T} P_{k} P_{k}^{T} e_{w}=\left(P_{k}^{T} e_{w}\right)^{T}\left(P_{k}^{T} e_{w}\right)=\sum_{(v, j) \in \mathcal{I}_{w}}\left(\sqrt{\frac{w_{j}}{k+1}}\right)^{2}=1
$$

so that (3.13) implies (3.11), and we have ${ }^{4} P_{k} P_{k}^{T}=I$.

To prove that $B_{A, k+1}$ is upper triangular, if $A$ is, we show that

$$
\left(P_{k}^{T} e_{w^{\prime}}\right)^{T}\left(B_{A, k} \otimes A\right)\left(P_{k}^{T} e_{w}\right)=0
$$

whenever $w^{\prime} \succ w$ in the lexicographic ordering. For any $(v, j) \in \mathcal{I}_{w}$, and $\left(v^{\prime}, j^{\prime}\right) \in$ $\mathcal{I}_{w^{\prime}}$, we have either $j^{\prime}>j$ or $v^{\prime} \succ v$. Consequently, we get either $e_{v^{\prime}}^{T} B_{A, k} e_{v}=0$ or $e_{j^{\prime}}^{T} A e_{j}=0$. But the left hand side of (3.15) is just

$$
\sum\left(e_{v^{\prime}} \otimes e_{j^{\prime}}\right)^{T}\left(B_{A, k} \otimes A\right)\left(e_{v} \otimes e_{j}\right)=\sum\left(e_{v^{\prime}}^{T} B_{A, k} e_{v}\right)\left(e_{j^{\prime}}^{T} A e_{j}\right)=0,
$$

where the sums are over $(v, j) \in \mathcal{I}_{w}$ and $\left(v^{\prime}, j^{\prime}\right) \in \mathcal{I}_{w^{\prime}}$, both.

Lower triangularity is inherited analogously.

We end the proof by showing that $B_{A^{*}, k}=\left[B_{A, k}\right]^{*}$. Let $\widetilde{P}_{1}=1$ and define $\widetilde{P}_{j}=P_{j-1}\left(\widetilde{P}_{j-1} \otimes I\right)$ for $j \geq 1$. Using $P_{j} P_{j}^{T}=I$ and induction it follows that $\widetilde{P}_{j} \widetilde{P}_{j}^{T}=I$. Using (3.12) recursively, we see that $B_{A, k}^{T} \widetilde{P}_{k}=\widetilde{P}_{k}\left(A^{T}\right)^{\otimes k}$ and hence $B_{A, k}=\widetilde{P}_{k} A^{\otimes k} \widetilde{P}_{k}^{T}$, with the same argument as for (3.13). Finally, $B_{A^{*}, k}=\widetilde{P}_{k}\left(A^{*}\right)^{\otimes k} \widetilde{P}_{k}^{T}=\left[\widetilde{P}_{k} A^{\otimes k} \widetilde{P}_{k}^{T}\right]^{*}=\left[B_{A, k}\right]^{*}$.

\section{Differential systems}

In the case of a differential equation, when looking for the invariant manifold in the form of the graph of a function $g$ we saw that $g$ has to satisfy

$$
\widetilde{\eta}(s, g(s))=D g(s) \eta(s, g(s)),
$$

\footnotetext{
${ }^{4}$ The scaling was chosen as to achieve this.
} 
with $g(0)=0, D g(0)=0$. Inserting expansions (3.2) and (3.3) into (3.16) we get

$$
\begin{aligned}
& \sum_{i+j \geq 1} \widetilde{\mu}_{i, j}[s]^{i}\left[\sum_{k \geq 2} \gamma_{k}[s]^{k}\right]^{j}= \\
& =\sum_{k \geq 2} k \gamma_{k}\left([s]^{k-1}, \sum_{i+j \geq 1} \mu_{i, j}[s]^{i}\left[\sum_{l \geq 2} \gamma_{l}[s]^{l}\right]^{j}\right),
\end{aligned}
$$

with $\mu_{0,1}=0$ and $\widetilde{\mu}_{1,0}=0$, and where $A=\mu_{1,0}$ and $\widetilde{A}=\widetilde{\mu}_{0,1}$ have spectra $\Lambda$ and $\widetilde{\Lambda}$, respectively. Write (3.17) as

$$
\begin{aligned}
& \widetilde{A}\left(\gamma_{2}[s]^{2}+\gamma_{3}[s]^{3}+\ldots\right)+\widetilde{\mu}_{2,0}[s]^{2}+\widetilde{\mu}_{1,1}\left(s, \gamma_{2}[s]^{2}+\ldots\right)+\ldots \\
= & 2 \gamma_{2}\left(s, A s+\mu_{2,0}[s]^{2}+\mu_{1,1}\left(s, \gamma_{2}[s]^{2}+\ldots\right)+\ldots\right)+ \\
& +3 \gamma_{3}\left([s]^{2}, A s+\ldots\right)+\ldots
\end{aligned}
$$

Comparison of the $k$-linear parts gives

$$
\begin{aligned}
& \widetilde{A} \gamma_{2}[s]^{2}+\widetilde{\mu}_{2,0}[s]^{2}=2 \gamma_{2}(s, A s), \\
& \widetilde{A} \gamma_{3}[s]^{3}+\widetilde{\mu}_{1,1}\left(s, \gamma_{2}[s]^{2}\right)+\widetilde{\mu}_{3,0}[s]^{3}=2 \gamma_{2}\left(s, \mu_{2,0}[s]^{2}\right)+3 \gamma_{3}\left([s]^{2}, A s\right),
\end{aligned}
$$

These equations can again be solved recursively: the equation for $\gamma_{k}$ is now of the form

$$
\widetilde{A} \gamma_{k}[s]^{k}-k \gamma_{k}\left([s]^{k-1}, A s\right)=\text { given . }
$$

With notation (3.7) we can write (3.18) as

$$
\widetilde{A} \Gamma_{k}-\Gamma_{k} \widehat{B}_{A, k}=\text { given }
$$

where $\widehat{B}_{A, k}^{T}$ is the matrix of the linear map $M_{A}(p)(s)=D p(s) A s$ with respect to the monomial basis. Again (3.19) is uniquely solvable if $\sigma(\widetilde{A}) \cap \sigma\left(\widehat{B}_{A, k}\right)=\emptyset$.

Lemma 3.3. If $\sigma(A)=\left\{\lambda_{1}, \ldots, \lambda_{d}\right\}$, then

$$
\sigma\left(\widehat{B}_{A, k}\right)=\sigma(A)^{+k}:=\left\{\sum_{i=1}^{d} j_{i} \lambda_{i}|| j \mid=k, j \in \mathbb{N}_{0}^{d}\right\}
$$

i.e., the set of all $k$-fold sums of elements of $\sigma(A)$.

For a more general result, see [BK98], Thm. 3.1.

Proof. Again, take first a diagonalizable $A=X \Lambda X^{-1}$. Then

$$
\begin{aligned}
M_{A}(p)(s) & =D p(s) X \Lambda X^{-1} s=D\left(L_{X}(p)\right)\left(X^{-1} s\right) \Lambda X^{-1} s \\
& =M_{\Lambda}\left(L_{X}(p)\right)\left(X^{-1} s\right)=\left(L_{X}^{-1} \circ M_{\Lambda} \circ L_{X}\right)(p)(s),
\end{aligned}
$$


so that $M_{A}$ is similar to $M_{\Lambda}$. Clearly

$$
\sigma\left(M_{\Lambda}\right)=\left\{k \lambda_{1},(k-1) \lambda_{1}+\lambda_{2}, \ldots, k \lambda_{d}\right\} .
$$

Hence (3.20) holds, for diagonalizable $A$, and the proof is completed by continuity.

Now the nonresonance condition is

$$
\sigma(\widetilde{A}) \cap \sigma(A)^{+j}=\emptyset, j=1, \ldots, k,
$$

under which the Taylor expansion of $g$ exists (formally) up to terms of order $k$. For example, in the case of (strong) stable manifold, $\sigma(A)$ contains all eigenvalues with negative real parts. Then (3.21) is satisfied for all $k$.

$\widehat{B}_{A, k}$ can, like $B_{A, k}$, be expressed explicitly via the elements of $A$.

Proposition 3.4. Let $A=\left(\alpha_{i j}\right) \in \mathbb{C}^{d \times d}$ and $v, w$ be multi-indices of order $k$. Then

$$
\left(\widehat{B}_{A, k}\right)_{w, v}= \begin{cases}\sum_{i=1}^{d} \alpha_{i i} w_{i}, & \text { if } v=w \\ \alpha_{i j} \sqrt{w_{i} v_{j}}, & \text { if } v-w=e_{i}-e_{j} \text { for } i \neq j \\ 0 & \text { otherwise. }\end{cases}
$$

Furthermore, $\widehat{B}_{A^{*}, k}=\left(\widehat{B}_{A, k}\right)^{*}$, and $\widehat{B}_{A, k}$ is upper (respectively lower) triangular if $A$ is. Each row (or column) of $\widehat{B}_{A, k}$ has at most $d^{2}-d+1$ non vanishing elements.

Proof. $M_{A}(1)=0$ and for any $|w| \geq 1$

$$
\begin{aligned}
M_{A}\left(s^{w}\right) & =D\left(s^{w}\right) A s=\sum_{i=1}^{d} \frac{\partial s^{w}}{\partial s_{i}}(A s)_{i} \\
& =\sum_{i=1}^{d} w_{i} s^{w-e_{i}} \sum_{j=1}^{d} \alpha_{i j} s_{j}=\sum_{i, j=1}^{d} w_{i} \alpha_{i j} s^{w+e_{j}-e_{i}} .
\end{aligned}
$$

So that for any multi-indices $0 \leq|v|=|w|=k$

$$
\frac{c_{v}}{c_{w}}\left(\widehat{B}_{A, k}^{T}\right)_{v, w}=\sum_{(i, j) \in\{1, \ldots, d\}^{2}: v+e_{i}=w+e_{j}} w_{i} \alpha_{i j} .
$$

The case $0=v-w=e_{j}-e_{i} \Leftrightarrow i=j$ corresponds to the diagonal element $\left(\widehat{B}_{A, k}\right)_{v, v}=\frac{c_{v}}{c_{v}} \sum_{i=1}^{d} w_{i} \alpha_{i i}=\sum_{i=1}^{d} w_{i} \alpha_{i i}$. In the case $0 \neq v-w=e_{j}-e_{i}$ the sum has just one term, and $c_{w} / c_{v}=\sqrt{v ! / w !}=\sqrt{v_{j} / w_{i}}$. For other $(w, v)$ the sum is empty. Summing up, each row or column of $\widehat{B}_{A, k}$ has at most $1+d(d-1)$ nonvanishing elements.

Since multi-indices are real, we get that symmetric, conjugated or diagonal $A$ implies symmetric, conjugate or diagonal $\widehat{B}_{A . k}$, respectively. Furthermore, if the 
monomials are ordered lexicographically, then $v+e_{i}=w+e_{j}$ and $v \prec w$ together imply $i>j$ by the definition of lexicographic ordering. Hence upper triangular $A$ implies $\left(\widehat{B}_{A, k}\right)_{w, v}=0$ for any $v \prec w$, i.e. $\widehat{B}_{A, k}$ is upper triangular. The lower triangular case is analogous.

\section{Solving Sylvester equations}

The Bartels-Stewart algorithm ([BS72]) for solving Sylvester the equation

$$
A X-X B=C
$$

is to transform $B$ into upper triangular form $T=Q^{-1} B Q$ (e.g. using the Schur decomposition), so that $A X Q-X Q T=C Q$, and solve the columns of $\widetilde{X}=\left[\begin{array}{lll}\widetilde{x}^{1} & \ldots & \widetilde{x}^{m}\end{array}\right]=X Q$ recursively from ordinary linear systems

$$
\left(A-t_{j, j} I\right) \widetilde{x}^{j}=\widetilde{c}^{j}+\sum_{i=1}^{j-1} t_{i, j} \widetilde{x}^{i},
$$

where $\left[\begin{array}{lll}\widetilde{c}^{1} & \ldots & \widetilde{c}^{m}\end{array}\right]=C Q$, and finally get $X=\widetilde{X} Q^{-1}$.

Now, to apply the Bartels-Stewart algorithm to the case of discrete systems, consider the Sylvester equations (3.8) for $k \geq 2$. There we need to transform $B_{A, k}$ into upper triagonal form. Beyn and Kleß noticed in [BK98] that just one Schur decomposition, namely that of $A$, is enough. Given the Schur decomposition $A=Q T Q^{*}$ we get, as in the proof of Lemma 3.1, that $L_{A}=L_{Q}^{-1} \circ L_{T} \circ L_{Q}$. Further, by Proposition 3.2 the matrix $B_{T, k}$ is upper triangular in the basis of lexicographically ordered monomials. Note also that $L_{Q}^{-1}=L_{Q^{-1}}=L_{Q^{*}}$, so that we have the similarity transformation

$$
B_{A, k}=B_{Q, k} B_{T, k} B_{Q^{*}, k}=B_{Q, k} B_{T, k} B_{Q, k}^{*},
$$

where the last equality follows from Proposition 3.2. We can then use the recursion (3.11) to build matrices $B_{T, k}$ and $B_{Q, k}$. We end up solving equations of type

$$
\left(\widetilde{A}-t_{j, j} I\right) \widetilde{\Gamma}_{k, j}=\text { given },
$$

where $t_{j, j}$ 's are the diagonal elements of $B_{T, k}$, i.e., elements of $\sigma(A)^{\cdot k}$.

Similar reduction is obtained easily in the case of differential systems as well, i.e., for equations (3.19). Assume $A=Q T Q^{*}$, then

$$
\widehat{B}_{T, k}=B_{Q, k} \widehat{B}_{T, k} B_{Q, k}^{*}
$$

where $\widehat{B}_{T, k}$ is upper triangular by Proposition 3.4. The proposition also gives an explicit formula for the matrix $\widehat{B}_{T, k}$.

\section{Preserving sparsity}

Assume that the dimension of the system is large and we are interested in a low dimensional invariant manifold, i.e., $d \ll n$. Further, suppose that $L=D \phi(p)$ 
is sparse. Then $\widetilde{A}=\widetilde{V}^{T} L \widetilde{U}$ is usually not sparse and the sparsity is lost in (3.24). In [BK98] the following bordering idea (see: [Gov95],[Kel87]) is used. Set $\widehat{\Gamma}_{k}=\widetilde{U} \widetilde{\Gamma}_{k}$. Then $V^{T} \widehat{\Gamma}_{k}=0$ and (3.24) is equivalent to

$$
\left[\begin{array}{cc}
L-t_{j, j} I & U \\
V^{T} & 0
\end{array}\right]\left[\begin{array}{c}
\widehat{\Gamma}_{k, j} \\
Y
\end{array}\right]=\left[\begin{array}{c}
\text { given } \\
0
\end{array}\right]
$$

where $Y=0$, necessarily, which can be used as a check of accuracy. Now the coefficient matrix is sparse and reasonable pivoting strategies preserve the sparsity. If a special software is used for equations $(L-\lambda I) x=b$, then the block elimination of Govaerts and Pryce ([GP93]) is useful.

Note that for (3.25) we need only the matrices $U$ and $V$, i.e., basis' for the low dimensional eigenspaces of $L$ and $L^{T}$, respectively.

\section{Numerical approach}

In evaluating the right-hand sides of (3.8) or (3.19) the approach of [BK98] needs the coefficients $\mu_{i, j}, \widetilde{\mu}_{i, j}$ of $\eta$ and $\widetilde{\eta}$. For these we need symbolic or numerical differentiation, which may turn costly. Also the code for assembling the righthand sides from these derivatives becomes easily rather complicated. Here we take an approach that avoids these.

We propose the following. The $k$ 'th order Taylor expansion of $g$ is

$$
g(s) \approx \sum_{j=2}^{k} \gamma_{j}[s]^{j}=\Gamma_{2} \widehat{s}^{2}+\Gamma_{3} \widehat{s}^{3}+\cdots+\Gamma_{k} \widehat{s}^{k}=\Gamma H(s)
$$

where

$$
\Gamma=\left[\begin{array}{llll}
\Gamma_{2} & \Gamma_{3} & \ldots & \Gamma_{k}
\end{array}\right] \quad \text { and } \quad H(s)=\left[\begin{array}{c}
\widehat{s}^{2} \\
\vdots \\
\widehat{s}^{k}
\end{array}\right] .
$$

Consider first the map case. Assume, for notational simplicity, that the coordinates have been chosen so that equilibrium is at the origin, i.e., $p=0$. Omitting higher order terms, we want to solve $\Gamma$ from equation (see (2.1))

$$
\begin{aligned}
& \Phi(\Gamma, s):= \\
& \widetilde{V}^{T} \phi(U s+\widetilde{U} \Gamma H(s))-\Gamma H\left(V^{T} \phi(U s+\widetilde{U} \Gamma H(s))\right)=0
\end{aligned}
$$

for all $s$ near the origin. The idea now is to evaluate (4.1) at a number of points $s_{1}, \ldots, s_{m}$, set up the corresponding nonlinear system for $\Gamma$, and solve it using the Newton's method.

Now $\Gamma \in \mathbb{R}^{(n-d) \times \kappa}$, where $\kappa=\sum_{j=2}^{k}\left(\begin{array}{c}j+d-1 \\ d-1\end{array}\right)=\left(\begin{array}{c}k+d \\ d\end{array}\right)-d-1$, and we need at least this number of points in order to have enough equations.

Write the system of equations in matrix form

$$
F(\Gamma, S)=\left[\Phi\left(\Gamma, s_{1}\right) \Phi\left(\Gamma, s_{2}\right) \ldots \Phi\left(\Gamma, s_{m}\right)\right]=0 .
$$


Then, in the Newton's method, we solve the corrections $\Delta \Gamma$ from the approximate linearization

$$
\widetilde{A} \Delta \Gamma H(S)-\Delta \Gamma H(A S)=-F(\Gamma, S),
$$

where $S=\left[\begin{array}{lll}s_{1} & \ldots & s_{m}\end{array}\right]$ and $H(S)=\left[\begin{array}{lll}H\left(s_{1}\right) \ldots H\left(s_{m}\right) & \ldots\end{array}\right.$. If $m=\kappa$ and if the points are such that $H(S)$ is invertible, we end up with a Sylvester equation

$$
\widetilde{A} \Delta \Gamma-\Delta \Gamma B=C(\Gamma),
$$

where $C(\Gamma)=-F(\Gamma, S) H(S)^{-1}$. We have

$$
H(A s)=\left[\begin{array}{ccc}
B_{A, 2} & & \\
& \ddots & \\
& & B_{A, k}
\end{array}\right] H(s),
$$

so that $B$ is readily formed as a block diagonal matrix, and further, using the Schur decomposition $A=Q T Q^{*}$, we get also the similarity transformation to triangular form:

$$
B=\left[\begin{array}{ccc}
B_{Q, 2} & & \\
& \ddots & \\
& & B_{Q, k}
\end{array}\right]\left[\begin{array}{lll}
B_{T, 2} & & \\
& \ddots & \\
& & B_{T, k}
\end{array}\right]\left[\begin{array}{lll}
B_{Q^{*}, 2} & & \\
& \ddots & \\
& & B_{Q^{*}, k}
\end{array}\right] .
$$

Matrix $H(S)$ is Vandermonde-like (is a part of a Vandermonde matrix if $d=1$ ), thus it is likely to be ill-conditioned. For obtaing $C(\Gamma)$ we may encounter difficulties. Hence we propose to take an overdetermined system: $m>\kappa$ and apply the following.

Lemma 4.1. Assume $H(S)$ has full rank and let $H(S)=\widehat{L} \widehat{Q}^{*}$ be an $L Q_{-}$ factorization, i.e., $\widehat{L}$ is square and lower triangular and the columns of $\widehat{Q}$ are orthonormal. Then the least squares solution ${ }^{5}$ of (4.2) is given by the solution of (4.3), where $B$ is as in (4.4) and $C(\Gamma)=-F(\Gamma, S) \widehat{Q} \widehat{L}^{-1}$.

We allow $H(S)$ to be complex, since in some cases we will use complex points.

Proof. Let $Y$ be such that $[\widehat{Q} Y]$ is unitary. Then

$$
\begin{aligned}
\| \widetilde{A} \Delta & \Gamma H(S)-\Delta \Gamma H(A S)+F(\Gamma, S) \|_{F}^{2} \\
& =\left\|\left(\widetilde{A} \Delta \Gamma \widehat{L} \widehat{Q}^{*}-\Delta \Gamma B \widehat{L} \widehat{Q}^{*}+F(\Gamma, S)\right)[\widehat{Q} Y]\right\|_{F}^{2} \\
& =\|[\widetilde{A} \Delta \Gamma \widehat{L}-\Delta \Gamma B \widehat{L}+F(\Gamma, S) \widehat{Q} \quad F(\Gamma, S) Y]\|_{F}^{2} \\
& =\|(\widetilde{A} \Delta \Gamma-\Delta \Gamma B-C(\Gamma)) \widehat{L}\|_{F}^{2}+\|F(\Gamma, S) Y\|_{F}^{2} .
\end{aligned}
$$

Since the second term of the last form does not depend on $\Delta \Gamma$, the minimum is obtained at the minimum of the first term, i.e., when $\Delta \Gamma$ is the solution of (4.3).

\footnotetext{
${ }^{5}$ The one that minimizes the Frobenius norm of the error.
} 
Since $B$ is block diagonal, equation (4.3) separates into $k-1$ Sylvester equations similar to (3.8)

$$
\widetilde{A} \Delta \Gamma_{j}-\Delta \Gamma_{j} B_{A, j}=C(\Gamma)_{j}, \quad j=2, \ldots, k,
$$

where $C(\Gamma)_{j}$ is the block of $C(\Gamma)$ correspoding to $\Gamma_{j}$. Hence, these equations can be solved independently (e.g. in parallel).

Now the (simplified) Newton's method is straightforward: Just evaluate $F(\Gamma, S)$, multiply it with $\widehat{Q} \widehat{L}^{-1}$ and solve the Sylvester equation corresponding to this right-hand side and get the update $\Delta \Gamma$ for $\Gamma$.

Note that, since we have an overdetermined system, this typically will not converge to $F(\Gamma, S)=0$. Hence, for convergence, we monitor $\|\Delta \Gamma\|$ and later judge the accuracy of $\Gamma$ by how well the approximate manifold is $\phi$-invariant.

For a differential equation $\dot{x}=f(x)$ near equilibrium $p=0$ we get similarly equations

$$
\begin{aligned}
& \Phi(\Gamma, s):= \\
& \widetilde{V}^{*} f(U s+\widetilde{U} \Gamma H(s))-\Gamma D H(s) V^{*} f(U s+\widetilde{U} \Gamma H(s))=0
\end{aligned}
$$

and

$$
\widetilde{A} \Delta \Gamma-\Delta \Gamma \widehat{B}=C(\Gamma)
$$

where $\widehat{B}=\left[\begin{array}{ccc}\widehat{B}_{A, 2} & & \\ & \ddots & \\ & & \widehat{B}_{A, k}\end{array}\right], \widehat{B}_{A, j}=B_{Q, j} \widehat{B}_{T, j} B_{Q, j}^{*}$ for $j=2, \ldots, k$, and $C(\Gamma)=$ $-F(\Gamma, S) \widehat{Q} \widehat{L}^{-1}$, corresponding to (4.1) and (4.3), respectively.

Remark 4.1. Several comments are now in order:

1. We don't need to compute any derivatives and we need not assemble the right-hand sides of (3.8), (3.19). The price we then pay for this is that we get a nonlinear system and we need to solve such Sylvester equations for every Newton step. However, the matrices $\widetilde{A}, B_{A, k}$ in these equations don't change, which brings us some savings.

2. The resulting code for the present approach is quite simple. Probably because of the complicated multilinear Sylvester approach in paper [BK98] the details were worked out and computations given only for second order expansions.

3. For this approach we need the matrices $V, U$ (low dimensional eigenspaces), one Schur decomposition of size $d \times d$, one QL-decomposition of size $m \times \kappa$, and approximatively number of iterations $\times \kappa$ solutions of linear equations of type $(\widetilde{A}-\lambda I) x=b$ with the same $\widetilde{A}$ of sixe $(n-d) \times(n-d)$. An interesting question is, how well iterative linear solvers could exploit this repetition. Here $\kappa=\left(\begin{array}{c}k+d \\ d\end{array}\right)$ and we typically take $\kappa<m<3 \kappa$. 
4. The system of equations is almost block triangular in the sense that $\Gamma_{k}$ has very small contribution to the equations of $\Gamma_{j}, j<k$. Hence it saves work to start with low degree (usually we take second degree) approximation and then increase the degree during the iteration.

5. In the case of sparse $L$ we can use bordering and suitable pivoting for (4.3) exactly the same way as described in Section 3.

6. $U, V, D \phi(p)$ need not be very accurate, if we include the term with $\Gamma_{1} s$ in the expansion. Further, if the equilibrium $p$ is only approximate, we can correct this with a constant term $\Gamma_{0}$.

7. The main concern in applying the present approach is accuracy. Usually we get good approximations up to orders $5-8$. For this we already need to apply the following:

(a) For good conditioning of $\widehat{L}$ we usually take an overdetermined system such that $m / \kappa \approx 1.3$.

(b) Also we take more coefficients than we really want, if we are interested in order $k_{0}$ approximation, in the computations we typically set $k \geq$ $k_{0}+2$. The purpose is that the effects of higher order terms would be eliminated from the low order coefficients.

Effects of these tunings will be illustrated in Example 5.1 below.

8. Assume the system is well defined also in the complex domain. Then we may evaluate the equation at complex points, consider e.g. a discrete complex torus, or more exactly,

$$
S=\left(r_{1} T_{k}\right) \times\left(r_{2} T_{k}\right) \times \cdots \times\left(r_{d} T_{k}\right)
$$

where $T_{k}=\left\{e^{2 \pi i \frac{j}{k+1}} \mid j=0, \ldots, k\right\}$, and $r=\left(r_{j}\right), r_{j}>0$, is a scaling. If we include all the monomials up to degree $k$, the columns of $H(S)$ become orthogonal. Then $H(S) H(S)^{*}=\widehat{L} \widehat{L}^{*}$ is diagonal, so that $\widehat{L}$ is. For a multi-index $|w| \leq k,\left(\widehat{L}^{*}\right)_{w, w}=c_{w} r^{w}$ so that $H(S)$ is well conditioned for reasonable choices of $r$, unlike in a typical case of real evaluation points. The cost is that the system (4.2) then becomes overdetermined $\kappa / m=$ $(1+k)^{d} /\left(\begin{array}{c}k+d \\ d\end{array}\right) \leq d$ ! fold. If the dimension of the invariant manifold, $d$, is large, this clearly becomes an obstacle.

\section{$5 \quad$ Numerical experiments}

Example 5.1. We consider first a simple example, for which we can solve the coefficients exactly using symbolic computation. Hence, in this example, we can 


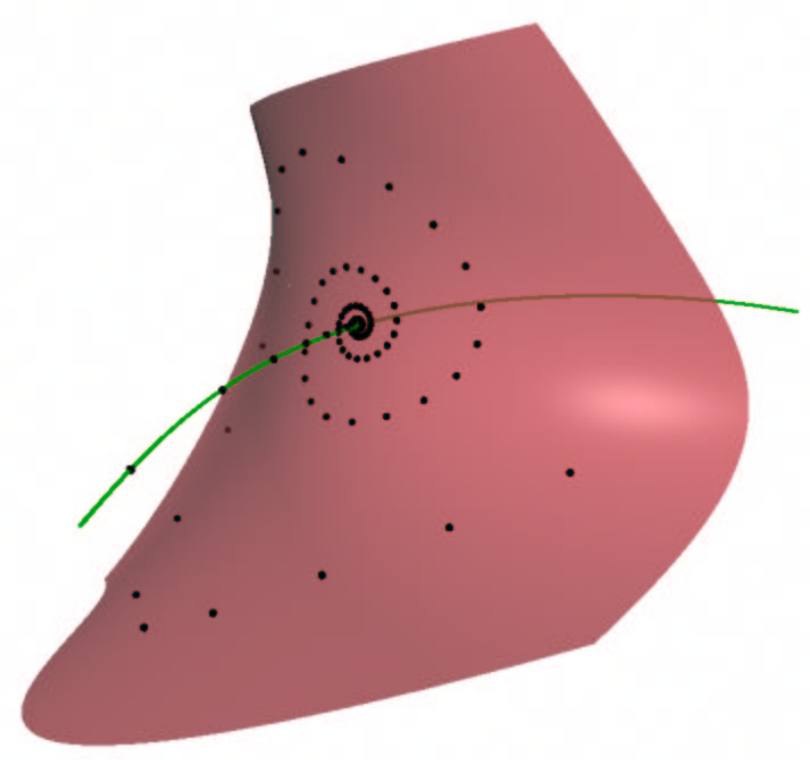

Figure 1: The stable and unstable manifolds of Example 5.1

monitor the true errors.

The origin is a hyperbolic equilibrium of the following map

$$
\phi(\boldsymbol{x})=\left[\begin{array}{c}
\frac{3}{5} x_{1}-x_{2} x_{3} \\
\frac{1}{5} x_{1}+x_{2}-\frac{3}{10} x_{3} \\
\frac{2}{5} x_{1}^{2}+\frac{2}{5} x_{2}+x_{3}
\end{array}\right] .
$$

It has the stable eigenvalue $\frac{3}{5}$ and a pair of complex unstable eigenvalues $1 \pm i \frac{\sqrt{3}}{5}$. Approximations of order six of the stable and unstable manifolds are drawn in Figure 1 together with a trajectory starting on the approximate stable manifold.

Here and in the later examples the Newton iteration converges usually in five steps.

In Figure 2 we illustrate points (7a), (7b) and (8) of Remark 4.1. On the vertical axis we show the logarithm of the errors of the coefficients $\Gamma_{j}, j=2, \ldots, 6$, when the approximation is of degree $k$. In the two figures on the top we have evaluated the equation on a star-like set

$$
\left\{\frac{1+(k \bmod \sqrt{m})}{\sqrt{m}}(\cos k / m, \sin k / m) \mid k=1, \ldots, m\right\} \subset \mathbb{R}^{2} .
$$

The number of points $m$ varies as the number of unknowns $\kappa$ varies with $k$, but $m / \kappa \approx 1.1$ and $m / \kappa \approx 3$ in respective figures. In the two lower figures the points are chosen uniformly from the complex torus in $\mathbb{C}^{2}$, as in Remark 4.1(8). The number of points corresponds here to $m / \kappa \approx 1.8$ and $m / \kappa \approx 3$.

The effect of increasing $k$ is clear. The effect of increasing $m / \kappa$ is not as clear, but it seems to allow the higher degree coefficients to be computed more accurately. 

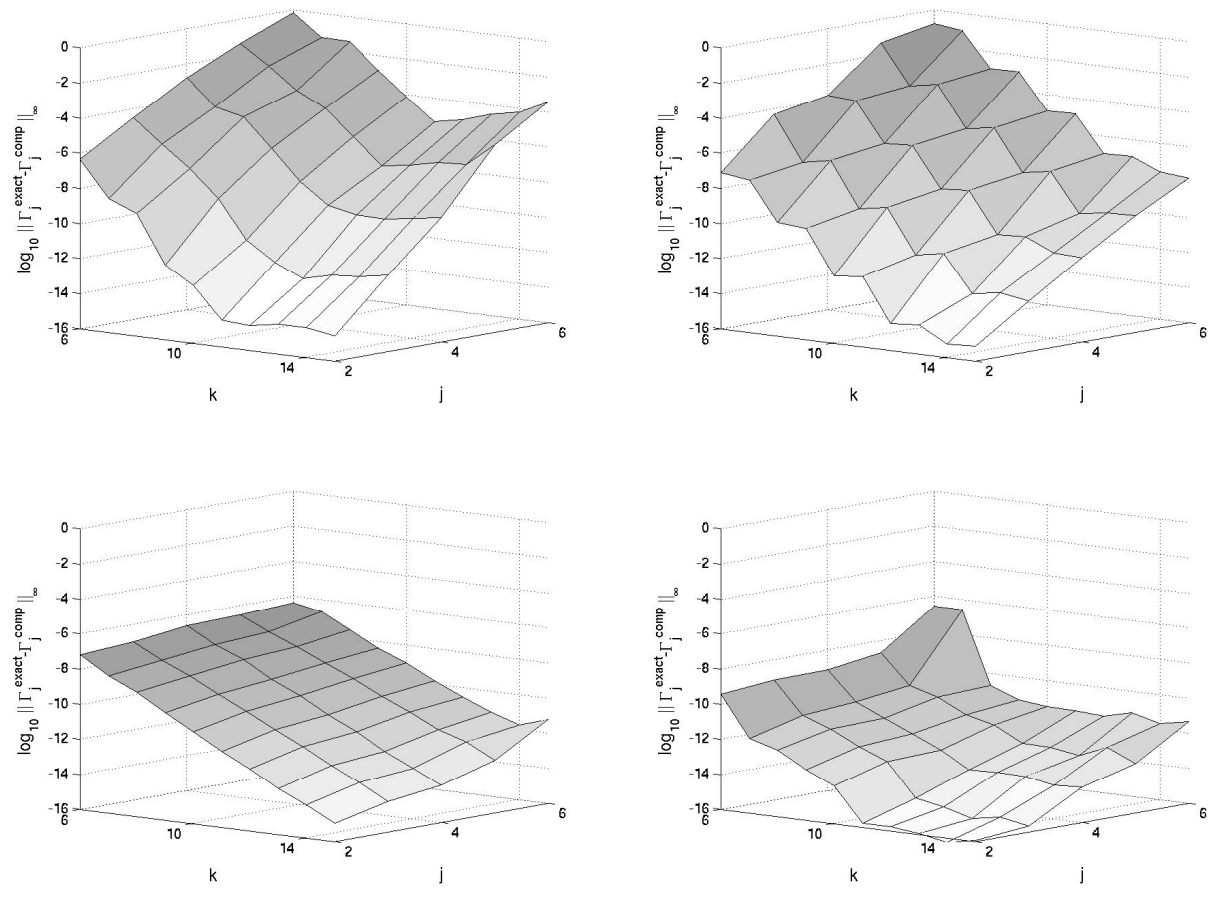

Figure 2: The figure is related to Example 5.1. In every subfigure we depict the accuracy of coefficients $\Gamma_{j}, j=2, \ldots, 6$, when the maximal degree of approximation $k$ used in the computation varies. The subfigures correspond to different choices of $S$. On the upper row $S$ is real and $m / \kappa \approx 1.1$, respectively, $m / \kappa \approx 3$. Below $S$ is subset of a complex torus and $m / \kappa \approx 1.8$ and $m / \kappa \approx 3$.

Choosing the complex evaluation points gives a drastic increase in the accuracy, though. Compare the second and fourth subfigure with both $m / \kappa \approx 3$.

Example 5.2. The coupled Tshebyshev maps ([Bec98],[Det]) are used as models for particle physics. They are maps of the $n$-cube $[-1,1]^{n}$ into itself, defined as

$$
\psi_{j}(x)=(1-a) f\left(x_{j}\right)+\frac{a}{2}\left[g\left(x_{j-1}\right)+g\left(x_{j+1}\right)\right],
$$

where $j=0,1, \ldots, n-1$ the indices are considered $\bmod n$, and functions $f$ and $g$ are Tshebyshev polynomials. Here we consider only the case $f(x)=T_{3}(x)=$ $4 x^{3}-3 x, g(x)=T_{1}(x)=x$.

The fixed points of $\psi$ are not interesting, but periodic trajectories are. The "in phase" periodic trajectories are those, for which $x^{k}=\mu_{k}\left[\begin{array}{l}1 \\ \vdots \\ 1\end{array}\right]$, where $\left\{\mu_{k}\right\}_{k \in \mathbb{Z}}$ is a periodic trajectory of $\mu_{k+1}=(1-a) f\left(\mu_{k}\right)+a g\left(\mu_{k}\right)$. Already two-periodic points, i.e., fixed points of $\phi(x)=\psi(\psi(x))$ show interesting behaviour. Such we get for

$$
\mu_{k}=(-1)^{k} \sqrt{\left(3-4 a-\sqrt{5-24 a+16 a^{2}}\right) /(8-8 a)} .
$$




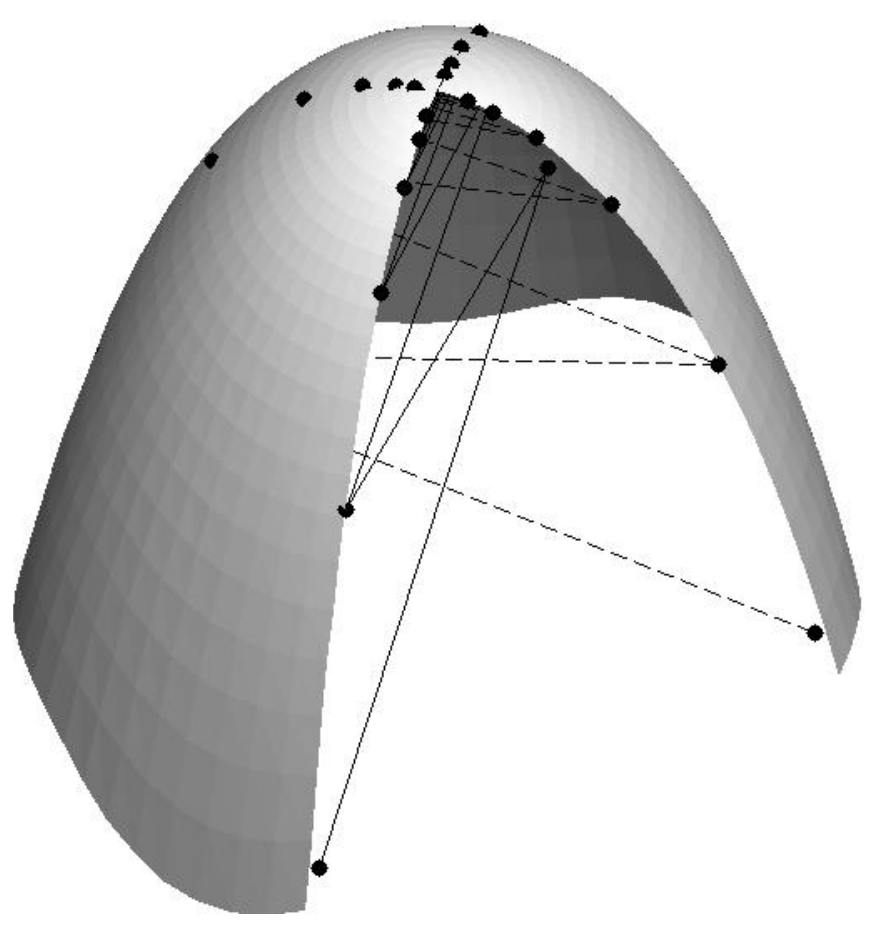

Figure 3: The local unstable manifold of the coupled Tshebyshev maps in Example 5.2 with two trajectories starting close to the fixed point. The points of the two trajectories are joined with a dashed, respectively, solid line. A quarter of the manifold is cut open.

Consider the case $n=5, a=0.2$, and $p=\mu_{0}\left[\begin{array}{l}1 \\ \vdots \\ 1\end{array}\right]$. The linearization has eigenvalues $-1.3451,-1.3451,-0.9649,-0.9649$ and -0.6800 , hence it has a two dimensional unstable manifold.

In the Figure 3 we have a sixth degree approximation of the local unstable manifold. The horizontal coordinates correspond to $U s$ and the vertical direction corresponds to the largest singular value of $\Gamma_{2}$. Also two trajectories of $\phi$ starting near $p$ are shown.

For this and later examples the natural test of accuracy is, how well the trajectories stay on the approximate manifold. We see that the outermost points are not exactly on the surface. This is due both to the low degree but also to the fact that we are approaching the convergence radius of the Taylor series (here the manifold is analytic).

Example 5.3. Consider the Lorenz system:

$$
\dot{x}=\left[\begin{array}{c}
\sigma\left(x_{2}-x_{1}\right) \\
\rho x_{1}-x_{1} x_{3}-x_{2} \\
x_{1} x_{2}-\beta x_{3}
\end{array}\right] .
$$

With parameter values $\sigma=10, \rho=28, \beta=8 / 3$, this has three equilibria

$$
p^{0}=(0,0,0), \quad p^{1}=(6 \sqrt{2}, 6 \sqrt{2}, 27), \quad p^{2}=(-6 \sqrt{2},-6 \sqrt{2}, 27) .
$$




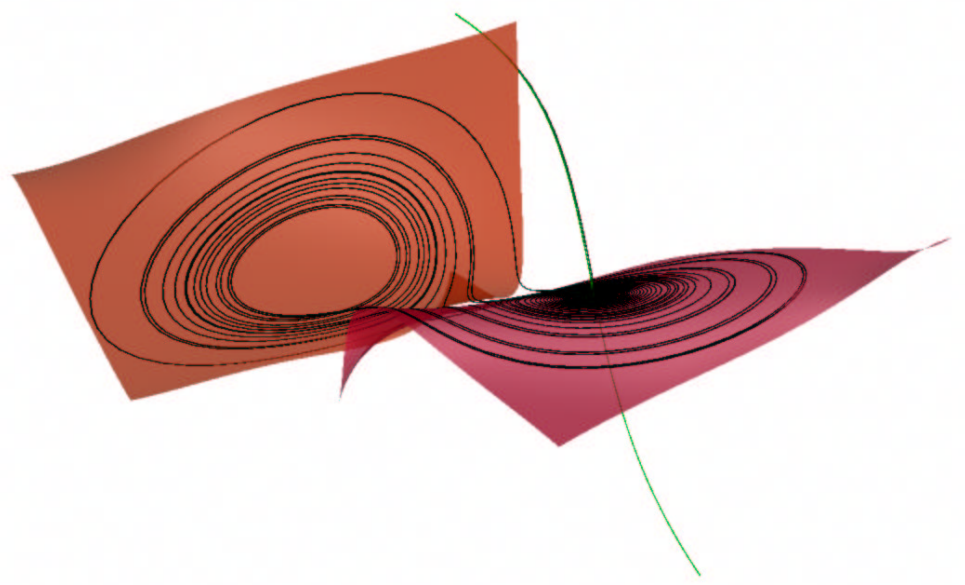

Figure 4: Lorenz system, Example 5.3.

The linearizations at $p^{1}$ and $p^{2}$ both have eigenvalues

$$
-13.8546 \text { and } 0.0939556 \pm 10.1945 i \text {. }
$$

Thus they have one-dimensional stable and two-dimensional unstable manifolds. In Figure 4, approximations of order eight of the stable and unstable manifolds of $p^{1}$ and the unstable manifold of $p^{2}$ are drawn together with a trajectory starting close to the stable manifold of $p^{1}$.

Example 5.4. The following coupled partial differential equation, was considered also in [BK98]:

$$
\left\{\begin{array}{l}
\dot{u}=\delta_{1} \Delta u+\lambda\left(a-(b+1) u+u^{2} v\right) \\
\dot{v}=\delta_{2} \Delta v+\lambda\left(b u-u^{2} v\right)
\end{array}\right.
$$

in one space dimension and with the Dirichlet boundary conditions $u=a, v=b / a$ at the end points. Clearly, these constants form an equilibrium solution of (5.3).

For the numerical computations we discretized system (5.3) using the standard three-point difference approximation for $\Delta$ with equally distributed grid points, $h=\frac{1}{50}(b-a)$, and fixed $2 \delta_{1}=\delta_{2}=0.0022, a=2$, and $b=6$.

The system of $(u, v)$ is known [BK98] to experience a Hopf bifurcation at $\lambda^{*}=$ $240(1-\cos (\pi / 200))$. We apply the Taylor expansion in $d=3$ variables to the discretized system (5.3) augmented with $\dot{\lambda}=0$ and obtain a three-dimensional system (2.3) approximating the Hopf bifurcation.

Performing the normal form computations (see, e.g.,[Kuz95]) we have

$$
\underline{\dot{s}}=(D \psi)(\underline{s}) \eta\left(\psi^{-1}(\underline{s}), g\left(\psi^{-1}(\underline{s})\right)\right) .
$$




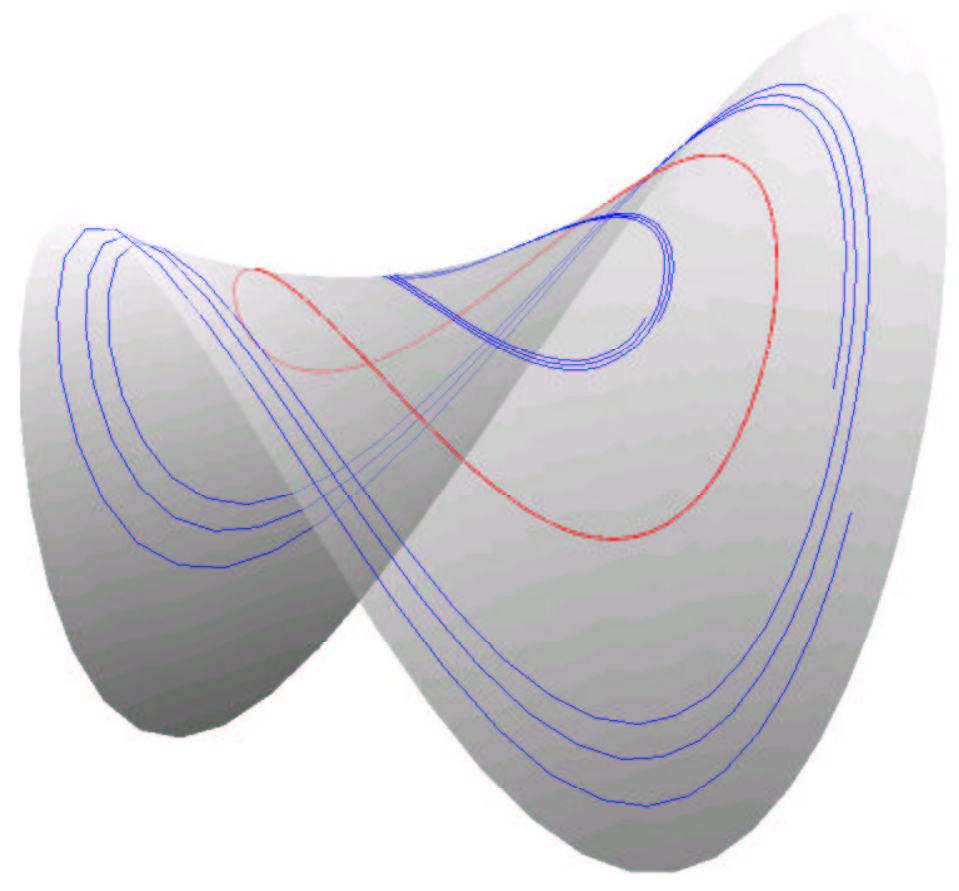

Figure 5: The invariant manifold $W$ of Example 5.4 with the periodic attractor and two other trajectories.

in new coordinates $\underline{s}=\psi(s ; \lambda)$, where $\psi$ is a polynomial (of degree three).

We evaluated the Taylor expansion around $\lambda_{0}=0.0300, u=a, v=b / a$, and further the normal form. From this we conluded that the critical value $\lambda^{*} \approx$ 0.0296082 , with accuracy of $10^{-15}$, and that the bifurcation is supercritical (a stable periodic solution appears for $\lambda>\lambda^{*}$ ). In comparison, using $\lambda_{0}=0.040$ we had $\lambda^{*} \approx 0.029620$, with an error of $\approx 10^{-5}$.

In Figure 5 a numerical approximation of the eigth degree Taylor expansion of the stable manifold for the equilibrium is shown together with three trajectories on $W$ in a neighbourghood of the equilibrium, here $\lambda=\lambda^{*}+0.001$. The horizontal coordinates correspond the projection of $U s$ to directions orthogonal to the $\lambda$ direction. The vertical axis corresponds to the largest singular value of $\Gamma_{2}$. The horizontal coordinates have been scaled to make the trajectory closer to a circle.

The three trajectories of $(5.3)$ are shown for $0 \leq t \leq 5 T_{\text {period. }}$ The inner and outer trajectories spiral towards the stable periodic solution. Here $T_{\text {period }} \approx 90.23$ is an estimator of the period of the periodic attractor is obtained from the normal form and it corresponds to the period of the computed trajectory with relative accuracy of 0.004 .

The distance $r_{\lambda}$ (in $E$-direction) of the periodic trajectory from the equlibrium is predicted by the normal form with relative accuracy of 0.005 .

In Figure 6 we show the vertical distance of the three numerically integrated 

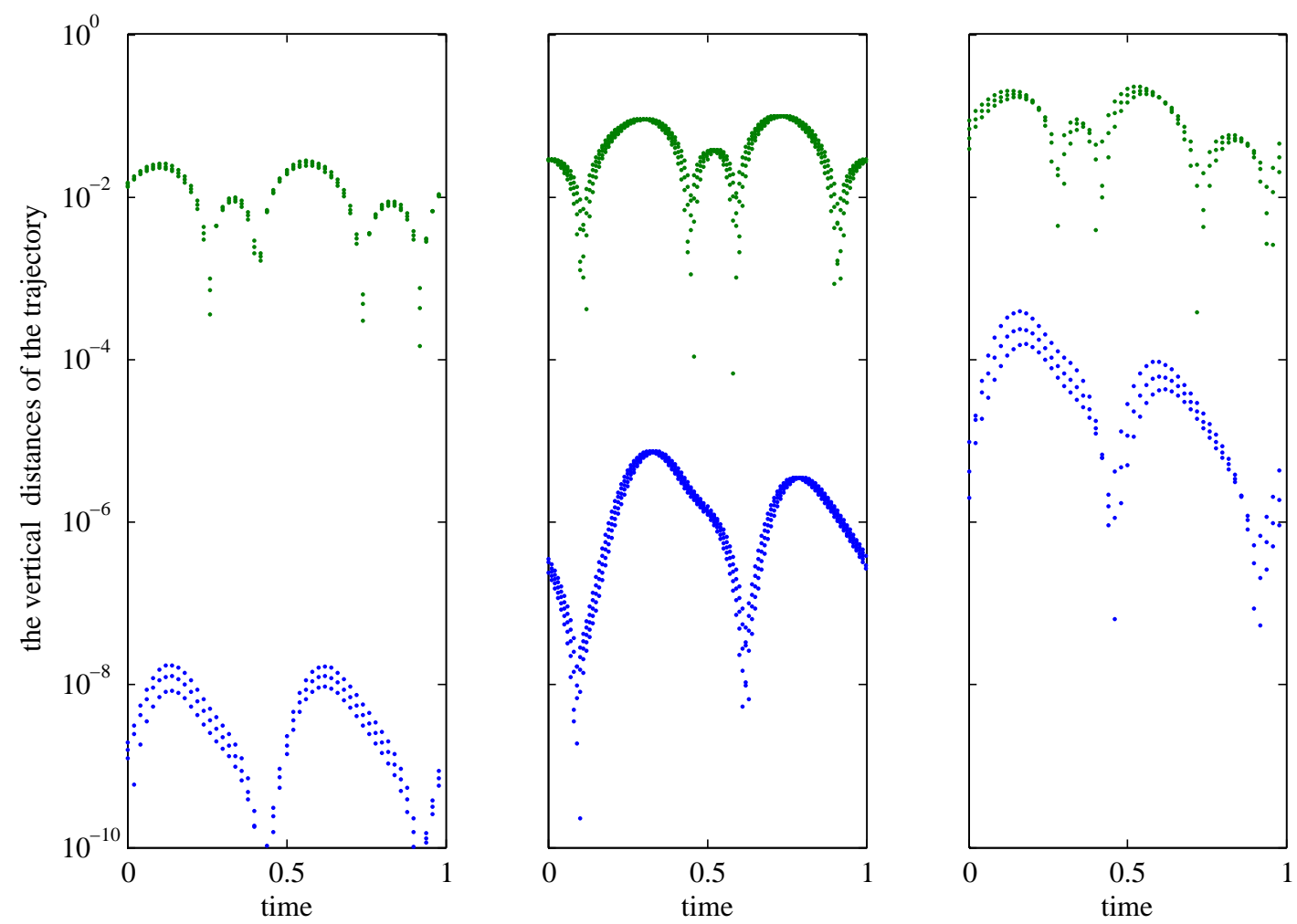

Figure 6: The vertical distance of each trajectory in Figure 5 to the computed manifold $W_{\text {comp }}$ (smaller values), and to $E$ (larger values). The horizontal axis corresponds to the time scaled by the computed period of the periodic trajectory, modulo one. The outer trajectory in Figure 5 corresponds to the right subfigure here.

trajectories to the manifold in Figure 5. The horizontal axis represents the time, the unit is relative to the computed period $T_{\text {period }}$ and modulo one. The slight distortion of the points in the time exhibits the difference of the computed period and the period of the computed trajectory. The distance in $E$ plane of the three trajectories from the equilibrium are $0.5,1$, and 1.5 times the distance of the periodic equlibrium. It is worth noticing, that the distance of the trajectory from the computed approximation of the manifold increases roughly $3^{8}$ fold as the distance from the equilibrium grows 3 fold.

We used Taylor expansion of degree 8 and also the normal form computations were performed up to the full degree 8. $S$ was chosen as in Remark 4.1.8, with $r=[20,20,2000]$.

Acknowledgement: The authors like to thank Olavi Nevanlinna for discussions and his suggestion to use complex evaluation points. 


\section{References}

[Bec98] C. Beck, Spontaneous symmetry breaking in a coupled map lattice simulation of quantized higgs fields, Phys. Lett. A 248 (1998), 386-392.

[BK98] W-J. Beyn and W. Kleß, Numerical Taylor expansions of invariant manifolds in large dynamical systems, Numer. Math. 80 (1998), 1-38.

[BS72] R. H. Bartels and G. W. Stewart, Solution of the matrix equation AX+ $X B=C$, CACM 15 (1972), 820-826.

[CH82] S-N. Chow and J. Hale, Methods of bifurcation theory, Springer-Verlag, New York, 1982.

[Det] C. P. Dettmann, Stable synchronised states of coupled tchebyscheff maps, Physica D.

[Gov95] W. Govaerts, Bordered matrices and singularities of large nonlinear systems, Int. J. Bifurcation and Chaos 5 (1995), 243-250.

[GP93] W. Govaerts and J. D. Pryce, Mixed block elimination for linear systems with wider borders, IMA J. Num. Anal. 13 (1993), 161-180.

[Har64] P. Hartman, Ordinary differential equations, John Wiley \& Sons, New York-London-Sydney, 1964.

[Kel87] H. Keller, Numerical methods in bifurcation problems, Springer Verlag, Bombay, 1987, Tata Institute of Fundamental Research.

[Kuz95] Y. A. Kuznetsov, Elements of applied bifurcation theory, Springer-Verlag, New York, 1995.

[Lan71] S. Lang, Algebra, Addison-Wesley, Reading, 1971.

[Shu87] M. Shub, Global stability of dynamical systems, Springer-Verlag, New York, 1987.

[Sim89] C. Simo, On the numerical and analytical appropximation of invariant manifolds, Les Methodes Modernes de la Mechanique Céleste (D. Benest and C. Froeschlé, eds.), Goutelas, 1989, pp. 285-329. 
(continued from the back cover)

A456 Ville Havu, Harri Hakula, Tomi Tuominen

A benchmark study of elliptic and hyperbolic shells of revolution

January 2003

A455 Yaroslav V. Kurylev, Matti Lassas, Erkki Somersalo

Maxwell's Equations with Scalar Impedance: Direct and Inverse Problems

January 2003

A454 Timo Eirola, Marko Huhtanen, Jan von Pfaler

Solution methods for R-linear problems in $C^{n}$

October 2002

A453 Marko Huhtanen

Aspects of nonnormality for iterative methods

September 2002

A452 Kalle Mikkola

Infinite-Dimensional Linear Systems, Optimal Control and Algebraic Riccati

Equations

October 2002

A451 Marko Huhtanen

Combining normality with the FFT techniques

September 2002

A450 Nikolai Yu. Bakaev

Resolvent estimates of elliptic differential and finite element operators in pairs of function spaces

August 2002

A449 Juhani Pitkäranta

Mathematical and historical reflections on the lowest order finite element models for thin structures

May 2002

A448 Teijo Arponen

Numerical solution and structural analysis of differential-algebraic equations

May 2002 
HELSINKI UNIVERSITY OF TECHNOLOGY INSTITUTE OF MATHEMATICS RESEARCH REPORTS

The list of reports is continued inside. Electronical versions of the reports are available at $h t t p: / / w w w . m a t h . h u t . f i / r e p o r t s /$.

\author{
A461 Tuomas Hytönen \\ Vector-valued wavelets and the Hardy space $H^{1}\left(R^{n} ; X\right)$ \\ April 2003 \\ A460 Jan von Pfaler, Timo Eirola \\ Numerical Taylor expansions for invariant manifolds \\ April 2003 \\ A459 Timo Salin \\ The quenching problem for the $\mathrm{N}$-dimensional ball \\ April 2003 \\ A458 Tuomas Hytönen \\ Translation-invariant Operators on Spaces of Vector-valued Functions \\ April 2003 \\ A457 Timo Salin \\ On a Refined Asymptotic Analysis for the Quenching Problem \\ March 2003
}

ISBN 951-22-6504-4

ISSN 0784-3143

HUT Mathematics, 2003 